\title{
IMT504: A New and Potent Adjuvant for Rabies Vaccines Permitting Significant Dose Sparing
}

\author{
Alejandro D. Montaner ${ }^{1}$, Analia De Nichilo ${ }^{1}$, Juan M. Rodriguez ${ }^{1}$, Andres Hernando-Insua ${ }^{1}$, \\ Juan Fló ${ }^{2}$, Ricardo A. Lopez ${ }^{2}$, Verónica Sierra ${ }^{3}$, Claudio Paolazzi ${ }^{3}$, Oscar Larghi ${ }^{1}$, \\ David L. Horn ${ }^{4 *}$, Jorge Zorzopulos ${ }^{2}$, Fernanda Elias ${ }^{1}$ \\ ${ }^{1}$ Instituto de Investigaciones Biomédicas, Fundación Pablo Cassará, Buenos Aires, Argentina; ${ }^{2}$ Immunotech S.A., Buenos Aires, \\ Argentina; ${ }^{3}$ Instituto San Jorge Bagó, Buenos Aires, Argentina; ${ }^{4}$ David Horn, LLC, Doylestown, USA. \\ Email: *B42181@aol.com
}

Received June $23^{\text {rd }}, 2012$; revised July $26^{\text {th }}, 2012$; accepted August $10^{\text {th }}, 2012$

\begin{abstract}
Background: Rabies virus infection causes encephalitis, which is almost always fatal. Vaccination can be extremely effective at preventing disease but is prohibitively costly. Vaccine formulations allowing dose-sparing and fewer inoculations with faster antibody response would be extremely desirable. IMT504, an immunostimulatory non-CpG oligodeoxynucleotide, is a highly potent vaccine adjuvant. Methods: Human and rat antibody measurements, and rat challenge studies were performed. Results: In rats, highly effective immune responses with IMT504 were observed even after diluting vaccine up to $1 / 625$. In highly lethal, live intracerebral rabies challenge studies, protection occurred even with extremely dilute vaccine plus IMT504. In humans, antibody titers developed faster and were significantly higher with IMT504-adjuvanted diluted vaccine vs non-adjuvanted vaccine (full strength or diluted). All five administered IMT504adjuvanted diluted vaccine reached protective antibodies $(\geq 0.5 \mathrm{IU} / \mathrm{ml})$ after the second injection. After the third injection, individuals receiving IMT504-adjuvanted diluted vaccine reached levels approximately 10 times higher than controls $(\mathrm{M} \pm \mathrm{SEM}$ : $31.0 \pm 10.9$ vs $3.40 \pm 0.99 \mathrm{IU} / \mathrm{ml})$. Conclusions: These data suggest that IMT504 may allow fewer inoculations, highly significant dose-sparing of vaccine, rapid antibody production and protection from rabies. Extensive clinical studies are necessary to confirm if the use of IMT504 will permit significantly greater access to highly effective life-saving rabies vaccines.
\end{abstract}

Keywords: IMT504; Adjuvant; Rabies; Vaccine; Human; Animal

\section{Introduction}

Rabies virus (RABV) is a negative sense single-stranded RNA virus with a typical bullet shape belonging to the genus Lyssavirus. RABV causes encephalitis in humans with a fatality rate of nearly $100 \%[1,2]$. Dog bites from rabid animals are the most common cause of infection in humans. The time period between exposure and the development of clinical rabies can be measured in months. However, pre-exposure vaccination or post-exposure rapid vaccination combined with rabies immune globulin are very effective at preventing encephalitis and death. The main correlate of protection post-vaccination is the presence of neutralizing antibody, while cytotoxic $\mathrm{T}$ cells do not appear to play a role in protection and may in fact be harmful to the host. Currently, rabies vaccines for humans, as recommended by the World Health Organization (WHO), consist of inactivated virus produced in

${ }^{*}$ Corresponding author. continuous cell lines [3]. Pre-exposure vaccination is administered in three intramuscular doses at 0,7 and 28 days. IgG antibody provides the most effective protection [4]. Post-exposure vaccination, as recommended by the $\mathrm{CDC}$, consists of accelerated administration of the vaccine over a short period of time. A standard course would be intramuscular inoculation at $0,3,7$, and 14 days [5]. Any rabies vaccine effective without the need for numerous re-inoculations and/or without the need for rabies immune globulin will significantly contribute to disease prevention and the number of deaths due to rabies by reducing the cost and complexity of pre and post-exposure prophylaxis.

Adjuvants are key components of vaccines in general, and immunostimulatory oligodeoxynucleotides (ODNs) are among the most promising adjuvants currently in development $[6,7]$. Immunostimulatory ODNs that are active on human cells are grouped into two major classes: 1) $\mathrm{CpG}$ ODNs, characterized by the presence of at least 
one active site bearing an unmethylated $\mathrm{CpG}$ in a given context [8], and 2) PyNTTTTGT ODNs that have at least one active site bearing the sequence PyNTTTTGT in which Py is C or T and N is A, T, C or G [9]. In vitro, among other effects, both kinds of ODNs induce expression of co-stimulatory molecules acting on plasmacytoid dendritic cells. However, phosphorothioate (PS) $\mathrm{CpG}$ ODNs induce the secretion of IFN alpha [8], while PS PyNTTTTGT ODNs do not [9], and PyNTTTTGT ODNs induce the release of GM-CSF acting on human NK and NKT cells in the presence of IL-2, while CpG ODNs do not (our unpublished data). Furthermore, PyNTTTTGT ODNs stimulate in vitro and in vivo the expansion of mesenchymal stem cell precursors while $\mathrm{CpG}$ ODNs do not [10]. Here, we report that IMT504, the prototype of the PyNTTTTGT class, provides a highly significant survival advantage after a lethal rabies challenge in a rat model, even with large dilutions of the rabies vaccine. In a pilot study performed in humans, IMT504-adjuvanted rabies vaccine also was very effective in boosting the overall antibody response using significantly less (1/5) antigen per dose.

\section{Materials and Methods}

\subsection{Animals}

Female Sprague-Dawley rats, 8 - 12 weeks old, were obtained from FUCAL Laboratories (Buenos Aires, Argentina) and housed in a facility at the School of Natural Sciences, University of Buenos Aires, Buenos Aires, Argentina. Animal care and use were according to international guidelines (Guide to the Care and Use of Experimental Animals. Canadian Council on Animal Care, 1998).

\subsection{Human Volunteers}

This study enrolled nine healthy male Caucasian volunteers who were naïve to rabies vaccine, age 39 to 51 years, with medium body frame and weight (range from 70.2 to $84.5 \mathrm{~kg}$ ). Volunteers signed an express informed consent form according to the International Ethical Guidelines for Biomedical Research Involving Human Subjects [11].

\subsection{Oligodeoxynucleotide IMT504}

For the rat studies, desalted PS ODN IMT504 (5'-TCATCATTTTGTCATTTTGTCATT-3') was synthesized with a wholly phosphorothioate backbone by Oligos ETC (Bethel, ME, USA). IMT504 was suspended in depyrogenated sterile saline, assayed for LPS contamination using the Limulus test, and kept at $-20^{\circ} \mathrm{C}$ until used. Purity was assessed by HPLC and PAGE assays. IMT504 was used if purity was $>97 \%$ and LPS levels were undetectable.

For the human studies, Vero cell RABV vaccine (Vero$\mathrm{Rab}^{\mathrm{TM}}$, Sanofi-Pasteur), a reduced dose $(0.1 \mathrm{~mL})$ was first mixed with IMT504 (1 mg dissolved in $0.2 \mathrm{~mL}$ sterile saline) and then diluted with sterile saline to have the same volume as the full dose, $(0.5 \mathrm{~mL})$. Control vaccine had $0.4 \mathrm{~mL}$ of sterile saline added to ensure all vaccines were of equal volume $(0.5 \mathrm{~mL})$ and appearance. Vaccines were administered intramuscularly in the deltoid mucle, according to the vaccine manufacturer's instructions.

The Department of Pharmacy, who were in no other way involved with the design or conduct of this study, were responsible for randomization of subjects and preparation of the injection solutions.

\subsection{Vaccines}

Vero cell RABV vaccine (VeroRab ${ }^{\mathrm{TM}}$, Sanofi-Pasteur) containing $\geq 2.5$ IU per dose was used for immunization of rats and humans. The initial pre-exposure human vaccination schedule recommended by the manufacturer consists of three intramuscular doses at days 0,7 and 21 or 28 , followed by a booster one year later [12].

\subsection{Immunization of Rats}

Each rat received a single intramuscular injection containing $1 / 5,1 / 25,1 / 125,1 / 625$ or $1 / 3125$ of the original vaccine. Control groups received the vaccine alone and experimental groups received vaccine with the addition of $50 \mu \mathrm{g}$ of ODN IMT504. Every two weeks up to day 60, the rats were bled and specific total $\mathrm{IgG}$ antibody was determined by ELISA.

\subsection{Immunization of Human Volunteers}

The rabies vaccine containing $1 / 5$ of the original amount of antigen, combined with saline or with $1 \mathrm{mg}$ of IMT 504 per dose was intramuscularly injected. Immunization consisted of three doses of the respective vaccine injected at days 0,30 and 60 .

\subsection{Antibody Determination in Rat Serum}

Specific IgG antibody in rat sera was determined by ELISA. Briefly, ELISA plates (Maxisorp ${ }^{\mathrm{TM}}$, Nunc) were coated overnight with $40 \mu \mathrm{g} / \mathrm{ml}$ of the recombinant rabies antigen. Residual protein-binding sites were blocked with carbonate-bicarbonate buffer containing $8 \%$ non-fat milk. Serum samples were diluted $1 / 200$ with $0.05 \%$ Tween 20 in phosphate buffered saline (PBS) and plates incubated $2 \mathrm{~h}$ at $37^{\circ} \mathrm{C}$. Total IgG was evaluated using Sigma A $5795 \mathrm{Ab}$ conjugated with horseradish peroxidase. Ophenylendiamine dichloride solution $(1 \mathrm{mg} / \mathrm{ml})$ was finally added to the wells. Optical Density (OD) was measured at $490 \mathrm{~nm}$. 


\subsection{Antibody Determination in Human Serum}

Specific anti-rabies human IgG antibodies were determined by an ELISA developed in our laboratory, as described above. Antibody responses were determined in serum samples diluted 1:100 at days $0,15,30,45,60,75$, 90 and 120 after the first vaccine injection.

Serum response to rabies vaccine alone or formulated with IMT504 was further determined by a commercial ELISA for the detection and titration of anti-rabies glycoprotein antibodies (PLATELIA ${ }^{\mathrm{TM}}$ Rabies II, Bio-Rad).

\subsection{Challenge Study in Rats}

The rabies virus challenge consisted of the Wistar rabies strain PM/WI 38-1503-3M passaged in Vero cells. Lethal dose (LD) $)_{50}$ for Sprague-Dawley rats was determined at the facilities of Instituto San Jorge Bagó (Buenos Aires, Argentina) as $1 \times 10^{6.65} / \mathrm{mL}$. The virus was conditioned to yield a challenge dose of $100 \mathrm{LD}_{50}$. Dilutions were made in sterile distilled water containing $2 \%$ horse serum. The intracerebral challenge was administered in $0.03 \mathrm{ml}$ on day 21 . The animals were observed twice a day for 21 days and rabies-related death or clinical signs were recorded. Deaths occurring within the first 24 hours of the challenge were judged not to be related to the virus and were excluded from the assay results.

Twelve groups of 10 animals each were studied. The assay was repeated three times. Each group received a given vaccine dilution $(1 / 5,1 / 25,1 / 125,1 / 625$ and $1 /$ 3125 ) with or without addition of $50 \mu \mathrm{g}$ of IMT504. Control groups were inoculated with saline with or without IMT504. Rabies virus-neutralizing antibodies were determined 21 days post inoculation (dpi).

\subsection{Statistical Analysis}

Statistical significance of differences was evaluated by the Student $t$-test. Differences were considered significant for $\mathrm{p}<0.05$.

\section{Results}

\subsection{Dose Response Analysis in Rats Injected with the Vero Cells Rabies Vaccine Plus IMT504}

Female Sprague-Dawley rats were intramuscularly injected with dilutions $(1 / 5,1 / 25,1 / 125,1 / 625)$ of the Vero cell rabies vaccine with or without addition of IMT504 as an adjuvant. Figure 1 shows mean and standard deviation of the rabies specific $\operatorname{IgG}$ at days 19, 35, 49 and 63 post-vaccination. The effect of the adjuvant can be clearly observed at the $1 / 125$ and $1 / 625$ dilutions. Particularly at the $1 / 125$ dilution, the mean IgG response in the group injected with vaccine plus IMT504 was larger than that obtained for any other group while in the corresponding group injected with the vaccine without addition of IMT 504, there was a marked decrease in IgG at all post-vaccination measurements.

\subsection{Challenge Study in Rats Immunized with the Vero Cell Rabies Vaccine Plus IMT504}

Vaccine potency of the Vero cell rabies vaccine with or without addition of IMT504 as an adjuvant was evaluated by a challenge assay using a virus concentration equivalent to $100 \mathrm{LD}_{50}$. Figure 2 shows that $100 \%$ protection was achieved with the IMT504-adjuvanted vaccine up to an antigen dilution of 1/625 while for the non-adjuvanted vaccine only antigen sparing up to a dilution of $1 / 25$ provided $100 \%$ protection.

The specific IgG antibody response was measured immediately before challenge (19 dpi). Figure 3 shows that this antibody response was similar for animals vaccinated with the vaccine plus IMT504 up to the dilution 1/625 in correlation with $100 \%$ protection up to this dilution in the challenge. In contrast, the specific IgG response was significantly decreasing from dilution $1 / 125$ in the animals injected with the non-adjuvanted vaccine. These data are in agreement with the observed increase in mortality with greater dilution.

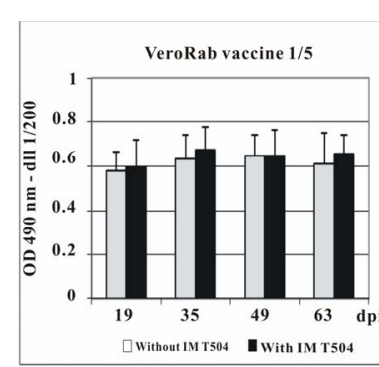

(a)

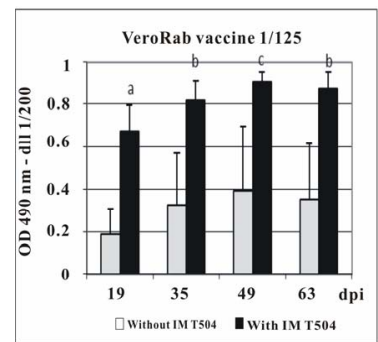

(c)

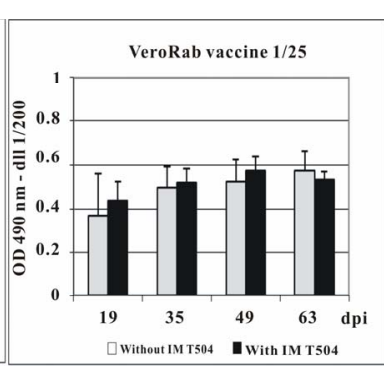

(b)

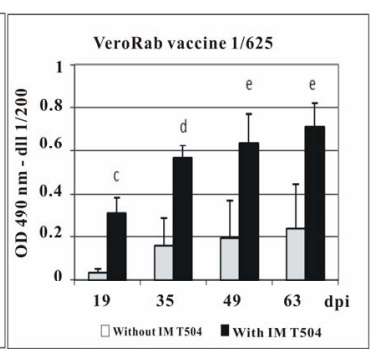

(d)
Figure 1. Antibody response in rats immunized with dilutions of Vero cell rabies vaccine plus IMT504. Animals (10 per group) were intramuscularly immunized with different dilutions of the Verorab $^{\text {TM }}$ (Sanofi-Pasteur) vaccine with or without addition of 50 ug of ODN IMT504. Each bar represents the median O.D. at $490 \mathrm{~nm}$ for specific total IgG as determined by ELISA. Statistically significant differences compared to controls (p); a: 0.001; b: 0.004; c: 0.008; d: 0.016; e: 0.003. dpi: days post inoculation. 


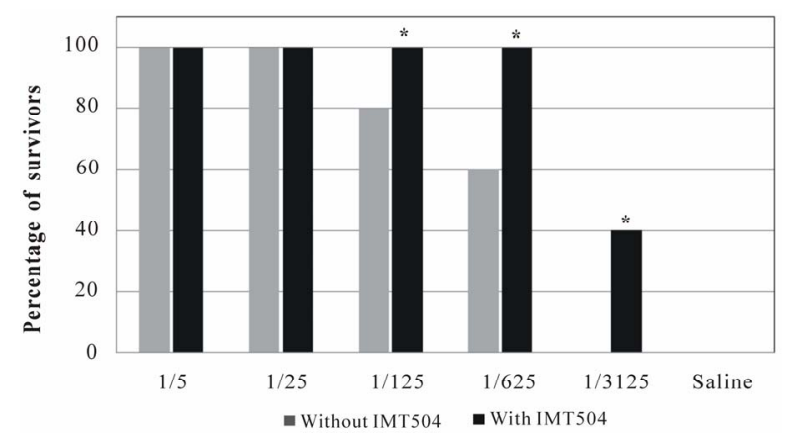

Figure 2. Challenge test in SD rats inoculated intra-cerebrally with $100 \mathrm{LD}_{50}$. Animals were immunized intramuscularly 21 days before virus challenge with different dilutions of the Verorab (Sanofi-Pasteur) vaccine with or without the addition of $50 \mathrm{ug}$ of ODN IMT504. Bars represent the survivor percentage up to day 21. Figure shows a representative experiment with 10 animals per group. Each test was performed three times. ( $\left.{ }^{*}\right)$ Asterisks indicate statistically significant differences $(p<0.01)$ compared to controls.

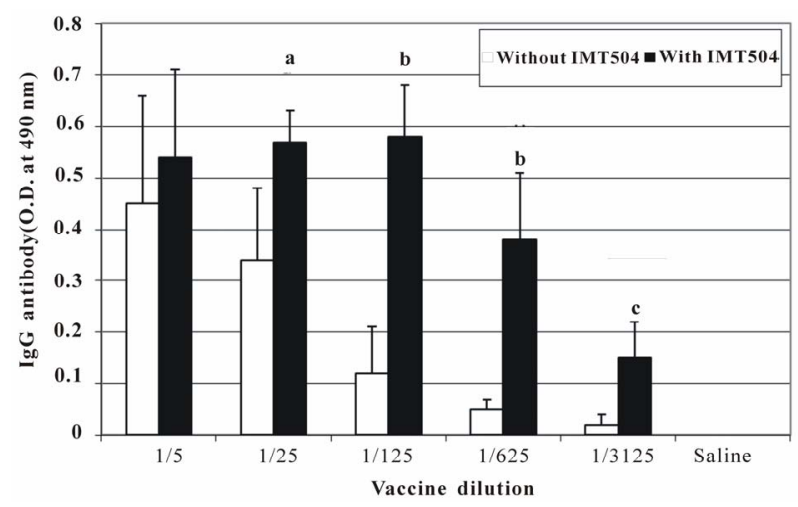

Figure 3. Antibody response from animals subjected to lethal intracranial rabies challenge. Total IgG response at 19 days post inoculation in animals subjected to challenge test. Each bar represents the median O.D. at $490 \mathrm{~nm}$ as determined by ELISA. Statistically significant differences compared to controls (p); a: 0.01 ; b: $<0.001$; : 0.02 .

\subsection{Immunization of Human Volunteers with Vero Cell Rabies Vaccine Containing 1/5 Dilution of Antigen Plus IMT504 as Adjuvant}

After reviewing the results obtained in rats that suggested a highly effective Vero cell rabies vaccine with a significant reduction in the antigen content due to the addition of IMT504, a pilot study in human volunteers was performed. A vaccine containing $1 / 5$ dilution of the original amount of antigen, with saline or IMT504 $(1 \mathrm{mg} /$ dose $)$ were intramuscularly injected in two groups of individuals naïve to rabies vaccination. Immunization consisted of three doses of the respective vaccine injected at days 0 , 30 and 60 . In order to assay the potency of the adjuvanted vaccine in humans using a significantly lower amount of antigen, rabies vaccine containing $1 / 5$ of the original amount of antigen, combined with saline or with $1 \mathrm{mg}$ of IMT504 per dose was intramuscularly injected. Immunization consisted of three doses of the respective vaccine injected at days 0,30 and 60 .

Even though a three dose regimen with inoculations at days 0,7 and 21 or 28 is currently recommended for vaccination with VeroRab ${ }^{\mathrm{TM}}$ [12], we chose a different schedule in which each injection was administered 30 days apart. We wanted to investigate the potential of the adjuvanted vaccine to be effective not only with a lower amount of antigen but also a lower number of injections. Therefore, 30 days was used in order to allow sufficient observation for each administered dose.

The immune response was assessed measuring the specific IgG in sera at days $0,15,30,45,60,75,90$ and 120 after the initial injection. Figure 4 reveals that the immune response measured as total antibody response was superior in the group of individuals injected with the IMT504-adjuvanted vaccine. Furthermore, anti-rabies glycoprotein antibodies were also measured using PLATELIA Rabies II ELISA (BioRad). Titers of anti-rabies glycoprotein antibodies have been reported to have a reasonable correlation with the titers of serum neutralizing antibodies measured by the rapid fluorescent focus inhibition test (RFFIT), which is currently accepted as the gold standard technique [13-15]. Table 1 shows that after the second injection, all of the individuals in the IMT504 group reached protective levels $(>0.5 \mathrm{IU} / \mathrm{ml})$ as defined by the WHO [3-16]. Notably, four out of five reached levels 60 - 90 times higher than the required protective level after the third dose. Antibody measurements were about 1 to 10 times the minimal protective amount in the group injected with the non-adjuvanted vaccine and about 2 to 50 times the minimal protective amount in the group injected with the IMT504-adjuvanted vaccine.

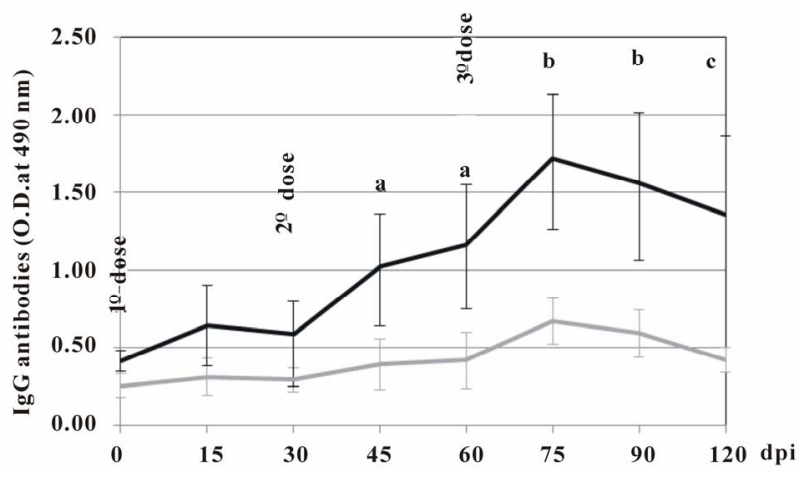

Figure 4. Evolution of antibody response in human volunteers immunized with 3 doses of the Vero cell rabies vaccine (Sa-nofi-Pasteur) diluted 1/5 and combined with saline or 1 mg of ODN IMT504. Statistically significant differences compared to controls (p); a: $0.04 ;$ b: 0.01 ; c: 0.02 . dpi: days post inoculation. 


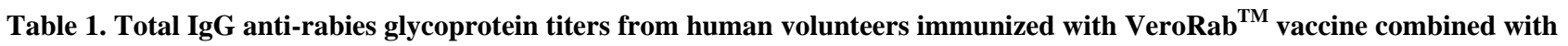
IMT504.

\begin{tabular}{cccccccccccc}
\hline & \multicolumn{4}{c}{ IMT504-adjuvanted group } & \multicolumn{5}{c}{ Control group } \\
\hline Time & E & F & G & H & I & A & B & C & D \\
15 days post 1st inoculation & $<0.1$ & $<0.1$ & $<0.1$ & $<0.1$ & $<0.1$ & $<0.1$ & $<0.1$ & $<0.1$ & 0.5 \\
15 days post 2nd inoculation & 1.60 & 22.60 & 13.60 & 21.00 & 26.50 & 2.23 & 0.94 & 0.47 & 6.07 \\
15 days post 3rd inoculation & 3.79 & 31.10 & 32.40 & 41.80 & 45.90 & 2.06 & 2.77 & 3.86 & 4.92 \\
\hline
\end{tabular}

Antibody titers expressed in IU/mL in human volunteers immunized with 3 doses of Vero cell rabies vaccine (VeroRab ${ }^{\mathrm{TM}}$; Sanofi-Pasteur) diluted $1 / 5$ and combined with saline or $1 \mathrm{mg}$ of IMT504. Protective levels as defined by WHO: $\geq 0.5 \mathrm{IU} / \mathrm{ml}$. Antibody titration was performed using the PLATELIA ${ }^{\text {TM }}$ (Bio-Rad) kit.

This is particularly significant according to the criteria established by the WHO, as the values of $\mathrm{IU} / \mathrm{ml}$ reached in the group injected with the IMT504-adjuvanted vaccine correspond to individuals with elevated seroconversion. Furthermore, according to the predictive model of Strady et al. [17], those individuals reaching titers of 30 $\mathrm{IU} / \mathrm{ml}$ after vaccination may need a booster only after 5 10 years as compared to the more frequent booster schedule currently recommended by the WHO.

In addition, three normal volunteers were immunized in parallel with the regular pre-exposure vaccination scheme ( 0,7 and 28 days) using the undiluted, non-adjuvanted VeroRab ${ }^{\mathrm{TM}}$ vaccine. After the third dose, antibody titers were $29.8,3.2$ and $1.59 \mathrm{IU} / \mathrm{ml}$, respectively. These titers are within the limits described in the literature in trials using the VeroRab ${ }^{\mathrm{TM}}$ vaccine after administration of three doses (see for example: Warrell et al. [18]).

\section{Discussion}

Pre-exposure rabies vaccination requires three inoculations of the inactivated vaccine, and the WHO recommends children in areas where rabies is endemic to be vaccinated [3]. However, vaccination costs against rabies virus can be highly prohibitive and severely limit the usage of this life-saving vaccine. Intradermal regimes of vaccinations have been developed and used in tropical countries in an effort to reduce vaccination costs [3-20]. Even though this new vaccination mode may reduce costs, it requires multiple visits to vaccination facilities and skilled practitioners in order to administer the vaccine. Therefore, further improvements are necessary in the formulation of currently available vaccines in order to lower costs and/or increase efficiency. Consequently, cost-efficient improvements in current vaccine formulations allowing dose sparing and fewer inoculations with faster antibody induction would be highly desirable for both pre-exposure and post-exposure vaccination.

In this study we have shown that vaccination with a Vero cell rabies vaccine plus IMT504 resulted in an ef- fective immune response as measured by the rabies virus neutralizing antibody response in rats and in humans using only a fraction (1/5) of the antigen currently used in the vaccine. In rats, the salutary effects of IMT504 were observed after a $1 / 125$ dilution of the human vaccine dose. At this dilution the anti-rabies virus IgG response of the non-adjuvanted vaccine significantly decreased while the response of the adjuvanted vaccine was maintained. This was also observed after a 1/625 dilution even though at this dilution a small decrease in the IMT 504-adjuvanted vaccine was observed suggesting that this dilution may be approaching the limit to maintain the full vaccine potency in the presence of IMT504.

Challenge studies were performed in order to investigate the neutralization capacity of the specific antibodies raised by vaccination with low antigen concentrations in the presence of IMT504. Neutralization curves paralleled the IgG curves indicating that the neutralization antibody proportion was maintained during vaccination with very low antigen doses in the presence of IMT504.

Small animal models may not represent the actual situation in humans [21]. In fact, IMT504 has been demonstrated to be immunostimulatory only in rats, monkeys and humans, with IMT504 being most active in humans [9-22]. Therefore, we decided to perform a pilot study of rabies vaccination in human volunteers. The results of this pilot study suggest the possibility that using IMT504 as an adjuvant may facilitate a faster and higher antibody production with significant vaccine dose sparing that can be achieved with fewer inoculations in humans. All of the five individuals vaccinated with IMT504-adjuvanted vaccine reached levels of anti-rabies glycoprotein antibodies well over the limit considered protective $(>0.5$ $\mathrm{IU} / \mathrm{ml}$ ) after the second injection. In addition, $4 / 5$ of these individuals reached levels higher than $30 \mathrm{IU} / \mathrm{ml}$. According to Strady et al. [17], these individuals may need a booster only after 5 - 10 years as compared to the more frequent booster schedule currently recommended by the WHO. Clearly, these encouraging results are greatly limited by the small number of individuals assayed in this 
pilot study, and extensive clinical trials should be performed in order to validate the possible use of IMT504 as adjuvant in a new rabies vaccine aimed to reduce economic costs. Regarding this, extensive toxicity studies performed in our laboratory have demonstrated that IMT 504 is a very safe compound even at doses hundred times greater than the amount proposed for vaccines. In addition, we have reported promising pre-clinical results in two other vaccines adjuvanted with IMT504 [22,23].

In summary, these data in humans and rats suggest that the use of IMT504 as an adjuvant may allow a significant reduction of dosing of the rabies vaccine concomitantly with fewer inoculations. These promising early results suggest the value of performing future clinical studies since a reduction of economic costs and a much broader access to life-saving rabies vaccines are highly desirable aims, especially in the less developed parts of the world.

\section{REFERENCES}

[1] N. Johnson, A. F. Cunningham and A. R. Fooks, "The Immune Response to Rabies Virus Infection and Vaccination," Vaccine, Vol. 28, No. 23, 2010, pp. 3896-3901. doi:10.1016/j.vaccine.2010.03.039

[2] M. J. Warrell and D. A. Warrel, "Rabies and Other Lyssavirus Diseases," Lancet, Vol. 363, No. 9413, 2004, pp. 959-969. doi:10.1016/S0140-6736(04)15792-9

[3] WHO, "WHO Position Paper on Rabies Vaccines," Weekly Epidemiol Record, Vol. 82, No. 49-50, 2007, pp. 425436.

[4] G. S. Turner, "Immunoglobulin (IgG) and (IgM) Antibody Responses to Rabies Vaccine," Journal of General Virology, Vol. 40, 1978, pp. 595-604. doi:10.1099/0022-1317-40-3-595

[5] Centers for Disease Control and Prevention, "Use of a Reduced (4-Dose) Vaccine Schedule for Postexposure Prophylaxis to Prevent Human Rabies," Morbidity and Mortality Weekly Report, Vol. 59, No. 2, 2010, pp. 1-9.

[6] H. L. Davis, R. Weeratna, T. J. Waldschmidt, L. Tygrett, J. Schorr, A. M. Krieg and R. Weeranta, "CpG DNA Is a Potent Enhancer of Specific Immunity in Mice Immunized with Recombinant Hepatitis B Surface Antigen," The Journal of Immunology, Vol. 160, No. 2, 1998, pp. 870-876.

[7] S. A. Halperin, G. Van Nest, B. Smith, S. Abtahi, H. Whiley and J. J. Eiden, "A Phase I Study of the Safety and Immunogenicity of Recombinant Hepatitis B Surface Antigen Co-Administered with an Immunostimulatory Phosphorothioate Oligonucleotide Adjuvant," Vaccine, Vol. 21, No. 19-20, 2003, pp. 2461-2467. doi:10.1016/S0264-410X(03)00045-8

[8] A. M. Krieg, "CpG Motifs in Bacterial DNA and Their Immune Effects," Annual Review of Immunology, Vol. 20, No. 1, 2002, pp. 709-760. doi:10.1146/annurev.immunol.20.100301.064842

[9] F. Elias, J. Flo, R. A. Lopez, J. Zorzopulos, A. Montaner and J. M. Rodriguez, "Strong Cytosineguanosine-Inde- pendent Immunostimulation in Humans and Other Primates by Synthetic Oligodeoxynucleotides with PyNTTTTGT Motifs," The Journal of Immunology, Vol. 171, No. 7, 2003, pp. 3697-3704.

[10] A. Hernando-Insua, A. D. Montaner, J. M. Rodriguez, F. Elias, J. Flo, R. A. Lopez, J. Zorzopulos, E. L. Hofer and N. A. Chasseing, "IMT504, the Prototype of the Immunostimulatory Oligonucleotides of the PyNTTTTGT Class, Increases the Number of Progenitors of Mesenchymal Stem Cells both in Vitro and in Vivo: Potential Use in Tissue Repair Therapy," Stem Cells, Vol. 25, No. 4, 2007, pp. 1047-1054. doi:10.1634/stemcells.2006-0479

[11] Council for International Organizations of Medical Sciences International, "Ethical Guidelines for Biomedical Research Involving Human Subjects," Bulletin of Medical Ethics, Vol. 182, 2002, pp. 17-23.

[12] http://drug.fda.moph.go.th/zone_search/files/VERORAB _1C\%20339_47.pdf

[13] M. Feyssaguet, L. Dacheux, L. Audry, A. Compoint, J. L. Morize, I. Blanchard and H. Bourhy, "Multicenter Comparative Study of a New ELISA, PLATELIA RABIES II, for the Detection and Titration of Anti-Rabies Glycoprotein Antibodies and Comparison with the rapid Fluorescent Focus Inhibition Test (RFFIT) on Human Samples from Vaccinated and Non-Vaccinated People," Vaccine, Vol. 25, No. 12, 2007, pp. 2244-2251. doi:10.1016/j.vaccine.2006.12.012

[14] R. J. Welch, B. L. Anderson and C. M. Litwin, "An Evaluation of Two Commercially Available ELISAs and One In-House Reference Laboratory ELISA for the Determination of Human Anti-Rabies Virus Antibodies," Journal of Medical Microbiology, Vol. 58, No. 6, 2009, pp. 806-810. doi:10.1099/jmm.0.006064-0

[15] S. M. Moore and C. A. Hanlon, "Rabies-Specific Antibodies: Measuring Surrogates of Protection against a Fatal Disease," PLoS Neglected Tropical Diseases, Vol. 4, 2010, pp. 1-6. doi:10.1371/journal.pntd.0000595

[16] S. A. Plotkin, "Vaccines: Correlates of Vaccine-Induced Immunity," Clinical Infectious Diseases, Vol. 47, No. 3, 2008, pp. 401-409. doi:10.1086/589862

[17] C. Strady, R. Jaussaud, I. Béguinot, M. Lienard and A. Strady, "Predictive Factors for the Neutralizing Antibody Response Following Pre-Exposure Rabies Immunization: Validation of a New Booster Dose Strategy," Vaccine, Vol. 18, No. 24, 2000, pp. 2661-2667. doi:10.1016/S0264-410X(00)00054-2

[18] M. J. Warrell, A. Riddell, L.-M. Yu, J. Phipps, L. Diggle, H. Bourhy, J. J. Deeks, A. R. Fooks, L. Audry, S. M. Brookes, F.-X. Meslin, R. Moxon, A. J. Pollard and D. A. Warrell, "A Simplified 4-Site Economic Intradermal Post-Exposure Rabies Vaccine Regimen: A Randomised Controlled Comparison with Standard Methods," PLoS Neglected Tropical Diseases, Vol. 2, No. 4, 2008, pp. 1-9. doi:10.1371/journal.pntd.0000224

[19] M. J. Warrell, P. Suntharasamai, K. G. Nicholson, D. A. Warrell, P. Chanthavanich, C. Viravan, A. Sinhaseni, R. Phanfung, C. Xueref and J. C. Vincent-Falquet, "MultiSite Intradermal and Multi-Site Subcutaneous Rabies Vaccination: Improved Economical Regimes," Lancet, Vol. 1, 
No. 8382,1984 , pp. $874-876$. doi:10.1016/S0140-6736(84)91340-0

[20] J. Lang, D. Q. Hoa, N. V. Gioi, N. C. Vien, C. V. Nguyen, N. Rouyrre and R. Forrat, "Immunogenicity and Safety of Low-Dose Intradermal Rabies Vaccination Given during an Expanded Programme on Immunization Session in Viet Nam: Results of a Comparative Randomized Trial," Transactions of the Royal Society of Tropical Medicine and Hygiene, Vol. 93, No. 2, 1999, pp. 208-213. doi:10.1016/S0035-9203(99)90309-7

[21] X. Wang, M. Bao, M. Wan, H. Wei, L. Wang, H. Yu, X. Zhang, Y. Yu and L. Wang, "A CpG Oligonucleotide Acts as a Potent Adjuvant for Inactivated Rabies Virus Vaccine," Vaccine, Vol. 26, No. 15, 2008, pp. 1893-1901. doi:10.1016/j.vaccine. 2008.01 .043
[22] F. Elias, J. Flo, J. M. Rodriguez, A. D. Nichilo, R. A. Lopez, J. Zorzopulos, C. Nagle, M. Lahoz and A. Montaner, "PyNTTTTGT Prototype Oligonucleotide IMT504 is a Potent Adjuvant for the Recombinant Hepatitis B Vaccine that Enhances the Th1 Response," Vaccine, Vol. 23, No. 27, 2005, pp. 3597-3603.

[23] A. D. Montaner, A. Denichilo, J. M. Rodríguez, J. Fló, R. A. López, A. Pontoriero, V. Savy, E. Baumeister, R. Frank, J. Zorzopulos and F. Elías, "Addition of the Immunostimulatory Oligonucleotide IMT504 to a Seasonal Flu Vaccine Increases Hemagglutinin Antibody Titers in Young Adult and Elder Rats, and Expands the Anti-Hemagglutinin Antibody Repertoire," Nucleic Acid Therapeutics, Vol. 21, No. 4, 2011, pp. 265-274. doi:10.1016/j.vaccine.2004.12.030 